\title{
Guest Editorial for the TOMACS Special Issue on the Principles of Advanced Discrete Simulation (PADS)
}

The Principles of Advanced Discrete Simulation (PADS) special issue is based on the selected papers from the 2017 ACM SIGSIM-PADS Conference, which is the flagship conference of the ACM's Special Interest Group on Simulation and Modeling (SIGSIM). Building on the 30 years of history and the reputation for high-quality papers, the 2017 ACM SIGSIM-PADS Conference was held at Nanyang Technological University, Singapore, on May 24-26, 2017. Philip Wilsey and Dong (Kevin) Jin are the guest editors of this special issue. They also served as the conference program chairs and were responsible for the proceedings of the conference.

The peer-review process for the special issue built on to the original reviews for the conference, wherein the submitted papers were reviewed by members of the SIGSIM-PADS International Program Committee, with papers receiving 3.67 referee reports on average and follow-on discussions. A total of 21 submissions were accepted and published as full papers and an additional 2 submissions were accepted and published as short papers in the proceedings. Of these, the authors of the top 6 papers selected by the guest editors together with the editor-in-chief of ACM Transactions on Modeling and Computer Simulation were invited to submit a substantially extended version of their work to the special issue. One of the invitees declined the invitation on the basis that a key member in the team left the original institution, so they were not able to produce sufficiently new results in time to meet the review schedule. The guest editors requested the authors to prepare a document outlining changes and extensions to their original ACM SIGSIM-PADS 2017 submission and that also took into account the reviewer comments received for the conference submission. The guest editors then considered the changes and requested clarification to the authors if required. During the review process, the referees were requested to check that the submitted special issue paper included the extensions and revisions that were planned by the authors.

The special issue papers were peer reviewed by a minimum of three reviewers. All underwent two rounds of reviews. One paper was rejected to the special issue in the second round of review. The special issue finally consists of four articles that are summarized below.

The first article (Rao and Higiro 2019) presents multi-tiered (two and three tier) data structures and a two-tier Ladder Queue data structure for both sequential and optimistic parallel simulations on distributed memory platforms, and the authors conduct an extensive evaluation of the proposed data structures based on 2,500 configurations of parallel hold (PHOLD) model and personal communication service (PCS) benchmarks. Results show that their data structures outperform a performance-tuned version of the Ladder Queue. The results also show that the three-tier heap and two-tier ladder queue outperform the Ladder Queue up to $60 \times$ in both sequential and optimistic parallel simulations. In addition, they conducted the same experimental analyses on two computing clusters to further ensure the simulation reproducibility.

The next article (Wu et al. 2019) presents a solution to address the load imbalance when simulating large-scale social contact networks with skewed topologies. They develop a hub location decomposition method to enhance the network partitioning as well as an agent decomposition method to reduce the communication overhead. They also investigate the off-loading and asynchronous stepping techniques to further reduce the impact caused by load imbalance during

(c) 2019 Association for Computing Machinery.

1049-3301/2019/03-ART8

https://doi.org/10.1145/3312749 
execution time. In addition, the authors develop an epidemic simulation and conduct extensive experiments to demonstrate that the proposed approach has significant performance improvement.

The third article (Linden et al. 2019) explores an approach for process synchronization of parallel simulation of spatially extended stochastic system models on multicore processors. The targeted mechanism is to provide effective synchronization support of processes with highly variable time intervals between successive events. The mechanism incorporates a mechanism for controlling optimism by having each process reports information about future outbound events. An implementation of the mechanism compared to an implementation with a naive synchronization shows a speedup of $43-95 \%$ for large models and an average speedup of $37 \%$ for small models.

The final article (Rahman et al. 2019) develops a hardware accelerator for parallel simulation. Specifically, the article describes an field-programmable gate array (FPGA) solution to manage object states and the event handling code. The solution specifically synthesizes the FPGA code to realize a custom hardware acceleration solution for a given simulation model. The accelerator is organized into multiple parallel event processors that can manage dependencies, record event execution history, and support rollback. The article examines an initial design configuration that scales to 64 concurrent event processors when additional scaling performance degrades. Using results from the initial solution, the article then evolves into a refined design solution with improved scaling capabilities. Comparing performance to a 12-core Intel Xeon processor, the article reports a 3.2 speedup factor that consumes $15 \%$ less power.

Preparations for the special issue began soon after the 2017 ACM SIGSIM Conference on PADS, and it has taken about 1.5 years for the articles to be ready for publication. A rigorous peer-review process has ensured that the selected papers represent not only the cutting edge of research in the PADS community but also provide future research direction and will, in time, contribute toward shaping the intellectual foundations of our discipline. The realization of the special issue was made possible through the contributions of numerous reviewers, and we thank them all. We are grateful for their time in preparing detailed referee reports and working to the timelines of the special issue.

\section{REFERENCES}

Jonatan Linden, Pavol Bauer, Stefan Engblom, and Bengt Jonsson. 2019. Exposing inter-process information for efficient parallel discrete event simulation of spatial stochastic systems. ACM Trans. Comput. Model. Simul. (2019).

Shafiur Rahman, Nael Abu-Ghazaleh, and Walid Najjar. 2019. PDES-A: A parallel discrete event simulation accelerator for FPGAs. ACM Trans. Comput. Model. Simul. (2019).

Dhananjai Rao and Julius Higiro. 2019. Managing pending events in sequential \& parallel simulations using three-tier heap and two-tier-ladder queue. ACM Trans. Comput. Model. Simul. (2019).

Yulin Wu, Wentong Cai, Zengxiang Li, Wen Tan, and Xiangting Hou. 2019. Efficient parallel simulation over large-scale social contact networks. ACM Trans. Comput. Model. Simul. (2019). 